Research Paper

\title{
Evolution of COVID-19 Disease Using a One Prey-Two Predator Model
}

\author{
Asini A. Konpola, ${ }^{a}$ Chathurika S. Gunasekara, a Indika G. K. \\ Udagedara, ${ }^{a}$ and Amila N. Sudu Ambegedaraa,
}

a Department of Mathematics, Faculty of Science, University of Peradeniya, Peradeniya 20400, Sri Lanka

Email correspondence: amilans@sci.pdn.ac.lk (A. N. S. Ambegedara)

Received: 30 March 2021; Revised: 23 April 2021; Accepted: 1 May 2021; Published: 31 May 2021

\begin{abstract}
Mathematical modeling is used to understand the dynamics of transmission of infectious diseases such as COVID-19, SARS, Ebola, and Dengue among populations. In this work, a one prey-two predator model has been developed to understand the underlying dynamics of COVID-19 disease transmission. We consider the infected, recovered, and death classes with the fact that an infected person can be transformed into the recovered or death group assuming that the infected ones are the prey, and the other two populations are the two predators in the one prey-two predator model. It was found that the proposed model has four equilibrium points; the vanishing equilibrium point (E_0), recovered and death-free equilibrium point (E_1), infected and recovered population-free equilibrium point (E_2), and the death-free equilibrium point (E_3). Stability analysis of the equilibrium points shows that all the other equilibrium points are locally stable. Global asymptotic stability of the recovered and death-free equilibrium point, infected and recovered free and death-free equilibrium point are also analyzed. Moreover, the existence and uniqueness of the solutions were proved. The parameters for the model are estimated from a data set that consists of the total number of infected, recovered, and death classes at a zone for the first 6 months of the year 2020 using the Nelder-Mead optimization method. During the first 6 months, the infected population increases at a higher rate, the recovered population, and the death class increase at a lower rate. However, some modifications to the system are needed. In future work, measures such as health precautions, vaccinations are needed to be considered to formulate the mathematical model and estimate parameters.
\end{abstract}

Keywords: Covid-19, local and global stability, mathematical modeling, parameter estimation 


\section{Introduction}

Mathematical modeling is used to understand the dynamics and behavior of the ecological interactions among the species such as prey and predator populations. Lotka and Volterra proposed the simplest model, which is known as the Lotka-Volterra model, of prey-predator interactions based on linear per capita growth rate [1]. Many fields like medicine [2], agriculture [3, 4] management, and social sciences [5] use mathematical modeling to model the dynamics of certain systems. Mathematical modeling can also be used to understand disease transmission among populations. Transmission of infectious diseases like COVID-19, SARS, Ebola, and Dengue can be easily understood using different mathematical models. In order to forecast disease outbreaks, avoid or cure these illnesses different mathematical models have been used in the literature $[2,6,7,8,9]$. Daniel Bernoulli in 1760 formulated and solved a model for the spread of smallpox in order to evaluate the effectiveness of variolation of healthy people with the smallpox virus [6]. In the 1920s, the emergence of compartmental diseases was taken place with the great work done by Kermack and McKendrick in 1927[7].

The world's deadly COVID-19 pandemic occurred in the ancient town of Wuhan Hubei Province, China, by the end of December 2019 spreading worldwide in 2020 and 2021. Initially, several unexplained cases of coughs, pneumonia, exhaustion, and fever were there among the people in Wuhan, China. The arrival of coronavirus resulted in the closure of businesses, schools, markets, traveling restrictions, curfew, lockdown, and reduction of gathering. With the arrival of SARS-CoV-2, the virus that causes COVID-19, researchers have been formulating mathematical models as a technique in getting an idea about the mode of transmission of the pandemic, impact of the pandemic, prevention, and control of the pandemic, the effect of the preventive measure on the pandemic [10,11-17]. Some recent developments of mathematical models and analysis are presented in [10], [16],[18]-[21],[23]-[37]. 
Traditionally, model validation is done by comparing the data with the model either qualitatively as stable or cyclic dynamic behavior, length of cycles, amplitudes, etc.... or quantitatively by estimating parameters in the field and calibrating the model to obtain a good fit for the data [38]. Fitting nonlinear models to time-series is a technique of increasing importance in population ecology [38], [39], [40]. In 2001, Jost and Arditi [38] applied this technique to assess the importance of predator dependence in the predation process by comparing two alternative models of equal complexity (one with and one without predator dependence) to predator-prey time series. Stochasticity in such data comes from both observation error and process error. They considered how these errors must be taken into account in the fitting process, and they developed eight different model selection criteria. Carpenter, Cottingham, and Stow in Ref.[39] fitted nonlinear difference equations to time series with observation errors, which were examined by stochastic simulation and analysis of plankton time series from two lakes. They showed that even modest observation errors cause errors in model identification and bias in parameter estimates and the latter problem can be corrected by estimation techniques that account for observation error, but model identification is difficult unless the state variables are manipulated. In Ref.[40], the authors applied model-fitting to test if typical ecological predator-prey time series data, which contain both observation error and process error, can give some information about the form of the functional response.

In this paper, a one prey-two predator model has been developed in such a way that the parameters of the model are found by using a set of COVID-19 data, which consists of the total number of infected people, recovered people, and dead population. The model is formulated to understand the dynamics of the spreading of COVID-19 data. The existence and uniqueness of the solutions were discussed and the conditions for the local and global asymptotic stability of the equilibrium points were established. 


\section{Materials and Methods}

\section{Model Formulation}

We consider a system consisting of three classes: infected, recovered, and dead due to COVID-19. The infected population is developed based on the logistic law with carrying capacity $K \geq 0$ and with the infection rate $a$. This can be happened due to the reasons such as immigration between zones and reported new Corona cases. Moreover, the model is formulated assuming that infected ones get converted to both recovered and dead classes. Similar to the Lotka-Volterra model formulation, it is assumed that the infected and recovered classes, and infected and death classes have meetings proportional to the product between the corresponding two classes. The recovered population can be increased or decreased due to the immigration between zones. The mathematical model as a system of three ordinary differential equations can be written as

$$
\begin{array}{cc}
\frac{d x}{d t}=a x\left(1-\frac{x}{K}\right)-d x y-c x z & \text { Equation 1 } \\
\frac{d y}{d t}=b y+d x y & \text { Equation 2 } \\
\frac{d z}{d t}=c x z, & \text { Equation 3 }
\end{array}
$$

where $x(0) \geq 0, y(0) \geq 0, z(0) \geq 0$. Here $x(t)$ is the infected population, $y(t)$ is the recovered population, and $z(t)$ is the dead class at the time $t$. However, for simplicity, these are represented as $x, y$, and $z$ in the text of this paper. In the model, the parameters represent the following. $a$ represents the growth rate of the infected population due to the factors such as traveling between zones and reported positive Corona cases, $K$ represents the carrying capacity of the infected population, $d$ is the cure rate, $c$ is the rate of transmission from infected to dead class, $b$ represents the rate of decrease or increase of recovered population due to the factors such as travelling between zones. The parameters $a$ and $b$ can take negative or positive values depending on the situation. 


\section{Parameter Estimation}

This section illustrates the parameter estimation method used to estimate parameters for the model. Let $p$ be the set of parameters of the systems of Ordinary Differential Equations. Then, the parameters can be obtained by minimizing the mean square error between the model output data $\boldsymbol{y}\left(t_{j} ; \boldsymbol{p}\right)$ and experimental or real data $\left(t_{j}, \boldsymbol{y}_{j}\right)$. Thus, the objective function that needs to be minimized is

$$
\operatorname{obj}(\boldsymbol{p})=\sum_{j=1}^{k}\left|\boldsymbol{y}\left(t_{j} ; \boldsymbol{p}\right)-\boldsymbol{y}_{j}\right|^{2} .
$$

In this work, the Nelder-Mead method is used to solve the above optimization problem [9]. The Nelder-Mead method, which was proposed by John Nelder and Roger Mead in 1965, is a numerical method that is used to find the minimum or maximum of an objective function in a higher-dimensional space. Algorithm 1 illustrates how the Nelder-Mead method works to find the optimal solution [10].

Algorithm 1: Nelder-Mead

1.Let $x_{i}$ denote the list of vertices in the current simplex, $i=1, \ldots, n+1$. 2.Order; Order and re-label the $n+1$ vertices from the lowest function value $f\left(x_{1}\right)$ to the highest function value $f\left(x_{n+1}\right)$ so that $f\left(x_{1}\right) \leq$ $f\left(x_{2}\right) \leq \cdots \leq f\left(x_{n+1}\right)$.

3.Reflect; Compute the reflection point $x_{r}$ by

$x_{r}=\bar{x}+\rho\left(\bar{x}-x_{(n+1)}\right)$,

where $\bar{x}$ is the centroid of the $n$ best points,

$\bar{x}=\sum\left(\frac{x_{i}}{n}\right), i=1, \ldots, n$.

If $f\left(x_{1}\right) \leq f\left(x_{r}\right)<f\left(x_{n}\right)$ then

replace $x_{n+1}$ with the reflected point $x_{r}$.

go to step 7 .

end if 


\section{Expand; \\ if $f\left(x_{r}\right)<f\left(x_{1}\right)$ then}

compute the expansion point $x_{e}$ by $x_{e}=\bar{x}+\chi\left(x_{r}-\bar{x}\right)$.

end if

if $f\left(x_{e}\right)<f\left(x_{r}\right)$ then

replace $x_{n+1}$ with $x_{e}$ and go to Step 7 .

else

replace $x_{n+1}$ with $x_{r}$ and go to Step 7 .

end if

\section{Constract;}

if $f\left(x_{r}\right) \geq f\left(x_{n}\right)$ then

perform a contraction between $\bar{x}$ and the better of $x_{n+1}$ and $x_{r}$. end if

\section{Outside contract;}

If $f\left(x_{n}\right) \leq f\left(x_{r}\right)<f\left(x_{n+1}\right)$ then

Calculate $x_{o c}=\bar{x}+\gamma\left(x_{r}-\bar{x}\right)$.

if $f\left(x_{o c}\right) \leq f\left(x_{r}\right)$ then

replace $x_{n+1}$ with $x_{o c}$

go to Step 7 .

else

go to Step 6.

end if

end if

\section{Inside contract;}

If $f\left(x_{r}\right) \geq f\left(x_{n+1}\right)$ then

Calculate $x_{i c}=\bar{x}+\gamma\left(x_{n+1}-\bar{x}\right)$

end if

if $f\left(x_{i c}\right) \geq f\left(x_{n+1}\right)$ then

replace $x_{n+1}$ with $x_{i c}$

go to Step 7 .

else

go to Step 6.

end if 
6.Shrink; Evaluate the $n$ new vertices

$x^{\prime}=x_{r}+\sigma\left(x_{i}-x_{1}\right), i=2, \ldots, n+1$.

Replace the vertices $x_{2}, \ldots, x_{+1}$ with the new vertices $x_{2}^{\prime}, \ldots, x_{n+1}^{\prime}$.

7.Stopping Condition; Order and re-label the vertices of the new simplex as $x_{1}, x_{2}, \ldots, x_{n+1}$ such that $f\left(x_{1}\right) \leq f\left(x_{2}\right) \ldots \leq f\left(x_{n+1}\right)$.

if $f\left(x_{n+1}\right)-f\left(x_{1}\right)<\varepsilon$ then

stop, where $\varepsilon>0$ is a small predetermined tolerance.

else

go to Step 3.

end if

\section{Results and Discussion}

\section{Population Dynamics}

Existence and Uniqueness of Solutions of the System

The existence and uniqueness of the system are proved using the existence and uniqueness theorem. The system of differential equations can be written in the following form and is analyzed by considering the continuity of the functions $f, g$ and $h$, which are the derivatives of $x, y$ and $z$ and the continuity of their partial derivatives with respect to $x$, $y$ and $z$ at the given initial conditions.

$$
\begin{aligned}
& \frac{d x}{d t}=f(x, y, z) \\
& \frac{d y}{d t}=g(x, y, z) \\
& \frac{d z}{d t}=h(x, y, z)
\end{aligned}
$$

\section{Equilibrium Points and Their Existence}

By setting right-hand sides of Equations 1, 2 and 3 to zero, four equilibrium points were found.

$$
\frac{d x}{d t}=0 \text { gives } a x\left(1-\frac{x}{K}\right)-d x y-c x z=0
$$


$\frac{d y}{d t}=0$ gives $b y+d x y=0$

Equation 5

$\frac{d y}{d t}=0$ gives $c x z=0$

Equation 6

By Equation 5, we can obtain $y=0$ or $x=-\frac{b}{d}$

By Equation 4, we can obtain $x=0$ or $a\left(1-\frac{x}{K}\right)-d y-c z=0$

By Equation 6, we can obtain $x=0$ or $z=0$

By considering $y=0$ and $z=0$ with $x=0$ one can obtain $(0,0,0)$ as a solution. Also, by considering $a\left(1-\frac{x}{K}\right)-d y-c z=0$ with $z=0$ and $y=0$ we can obtain $(K, 0,0)$ as the next solution. Also, by taking $a\left(1-\frac{x}{K}\right)-d y-c z=0, y=0$ and $x=0$ into account,

$\left(0,0, \frac{a}{c}\right)$ can be obtained as the solution. Finally, when $x=-\frac{b}{d}$ and $z=$ 0 , we can obtain $\left(-\frac{b}{d}, \frac{a}{d}\left(1+\frac{b}{d K}\right), 0\right)$ as a solution.

The four equilibrium points are summarized following.

1. The vanishing equilibrium point, $E_{0}=(0,0,0)$, always exist.

2. The recovered and death-free equilibrium point, $E_{1}=$ $(\tilde{x}, 0,0)$, where $\tilde{x}=K$, always exist.

3. The infected and recovered-free equilibrium point, $E_{2}=$ $(0,0, \hat{z})$, where and $\hat{z}=\frac{a}{c}$, exists provided that $a>c$.

4. The death free equilibrium point can be defined as $E_{3}=(\breve{x}, \breve{y}, 0)$, where $\breve{x}=-\frac{b}{d}$ and $\breve{y}=\frac{a}{d}\left(1+\frac{b}{d K}\right)$. Provided that $b<d$ and $\frac{a}{d}(1+$ $\left.\frac{b}{d K}\right)>0$.

\section{Local Stability Analysis}

The local asymptotic stability of the system of Equations 1, 2 and 3 at each equilibrium point is studied by computing the Jacobian matrix and finding the eigenvalues evaluated at each equilibrium point. For stability of the equilibrium points, the real parts of the eigenvalues of the Jacobian matrix evaluated at each equilibrium point must be negative. Thus, the 
conditions for which each equilibrium point to be locally asymptotically stable were found. Jacobian matrix for the system is:

$$
J(x, y, x)=\left(\begin{array}{ccc}
a-\frac{2 a x}{k}-d y-e z & -d x & -e x \\
f y & -b+f x & 0 \\
g z & 0 & -c+g x
\end{array}\right)
$$

Jacobian matrix at each equilibrium point is evaluated and the following results were obtained,

1. E0 is locally asymptotically unstable since $a>0$ always.

$$
J\left(E_{0}\right)=\left(\begin{array}{ccc}
a & 0 & 0 \\
0 & -b & 0 \\
0 & 0 & -c
\end{array}\right)
$$

2. $E_{1}$ is locally asymptotically stable if $f k<b$ and $g k<c$

$$
J\left(E_{1}\right)=\left(\begin{array}{ccc}
-a & -d k & -e k \\
0 & -b+f k & 0 \\
0 & 0 & -c+g k
\end{array}\right)
$$

a. Eigenvalues are $-a,-b+k f$ and $-c+g k$

3. $E_{2}$ is locally asymptotically stable if $\frac{c f}{g}<b$ and $g k<c$ by applying Routh's Criterion.

$$
J\left(E_{2}\right)=\left(\begin{array}{ccc}
-\frac{a c}{g k} & -\frac{d c}{g} & -\frac{e c}{g} \\
0 & -b+\frac{f c}{g} & 0 \\
\frac{g a}{e}\left(1-\frac{c}{g k}\right) & 0 & 0
\end{array}\right)
$$

4. By applying the Routh's criterion [12], E3 is locally asymptotically stable if $b<k f$ and $g b<c f$. 


\section{Global Stability Analysis}

The global stability behavior of the system of equations at the coexistent equilibrium point is investigated using the Lyapunov stability theorem [41]. By showing that the time derivative of the selected Lyapunov function is negative definite, the global asymptotic stability of the equilibrium points $E_{1}, E_{2}$, and $E_{3}$ were analyzed. The time derivative of the selected Lyapunov function was negative definite under some conditions for both the above equilibrium points. The selected Lyapunov function is,

$V(x, y, z)=P\left(x-x^{*}-x^{*} \ln \frac{x}{x^{*}}\right)+\left(y-y^{*}-y^{*} \ln \frac{y}{y^{*}}\right)+Q\left(z-z^{*}-z^{*} \ln \frac{z}{z^{*}}\right)$

where $P$ and $Q$ are arbitrary constants. By finding the time derivative of $V$ and substituting $P=1$ and $Q=\frac{1}{c}$ following equation is obtained.

$$
\frac{d v}{d t}=\frac{-a\left(x-x^{*}\right)^{2}}{K}+\left(z-z^{*}\right)\left(x(1-c)+c x^{*}\right) \quad \text { Equation } 7
$$

For equilibrium point $E_{1}, y^{*}=0$ and $z^{*}=0$ therefore,

$$
\frac{d v}{d t}=\frac{-a\left(x-x^{*}\right)^{2}}{K}+z\left(x(1-c)+c x^{*}\right)
$$

Equation 8

For equilibrium point $E_{2}, x^{*}=0$ and $y^{*}=0$ therefore,

$$
\frac{d v}{d t}=\frac{-a x^{2}}{K}+\left(z-z^{*}\right) x(1-c)
$$

For equilibrium point $E_{3}, z^{*}=0$, therefore,

$$
\frac{d v}{d t}=\frac{-a x\left(x-x^{*}\right)^{2}}{K}+z\left(x(1-c)+c x^{*}\right)
$$


Time derivative given by Equation 8 is negative definite if $c x^{*}<x(c-$ $1)$. Therefore, $E_{1}$ is globally asymptotically stable under the above condition. The time derivative given by Equation 9 is negative definite if one of the following conditions holds.

1. $z<z^{*}$ and $1<c$ or

2. $z>z^{*}$ and $1>c$

Therefore, $E_{2}$ is globally asymptotically stable under any one of the above two conditions. Time derivative given by Equation 10 is negative definite if $c x^{*}<x(c-1)$. Therefore, $E_{3}$ is globally asymptotically stable under above condition.

\section{Parameter Estimation}

In order to estimate the parameters, the Nelder-Mead method is used and data from https://datahub.io/core/covid-19\#resource-time-series-19covid-combined were used. Here we assumed that the individuals in the recovered population do not travel out of the zone. Then, the estimated parameters of the model are,

$$
a=1, K=200000, b=0, c=0.0007, d=0.001
$$

Figures 1, 2, and 3 show the time series evaluation of the infected, recovered, and death classes. One can see from the model that if the COVID-19 disease exists in society then the infected population is growing due to the slow recovery process and the low number of deaths. The recovered population also blowing up at a lower rate. It can be observed that the death class also increases at a lower rate within the 6 months period. Note that health precautions and vaccinations were not considered in the selected dataset. 


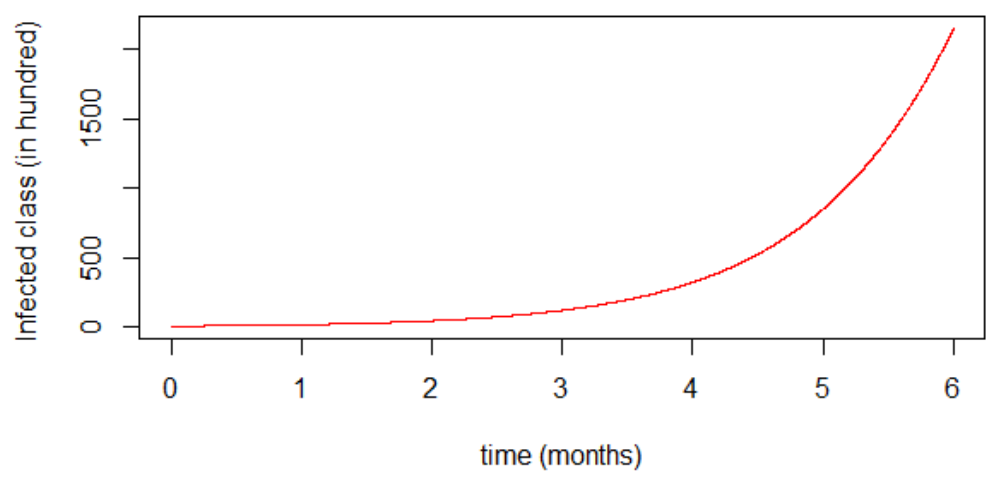

Figure 1.Variation of infected class with time.

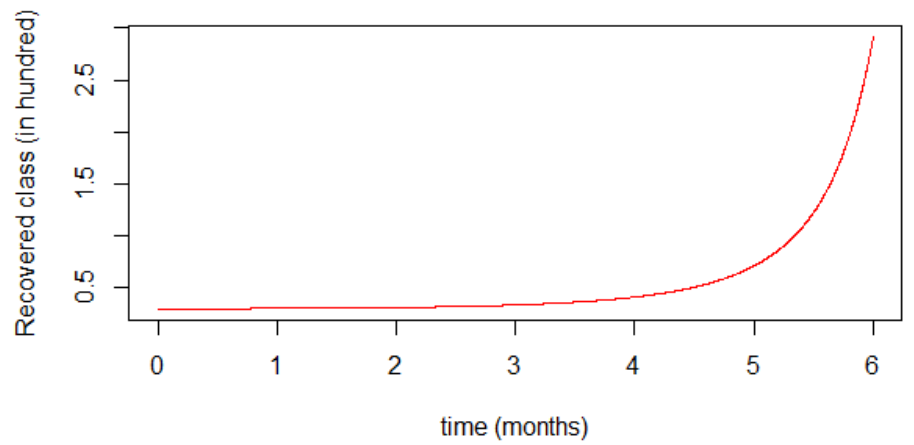

Figure 2.Variation of recovered class with time.

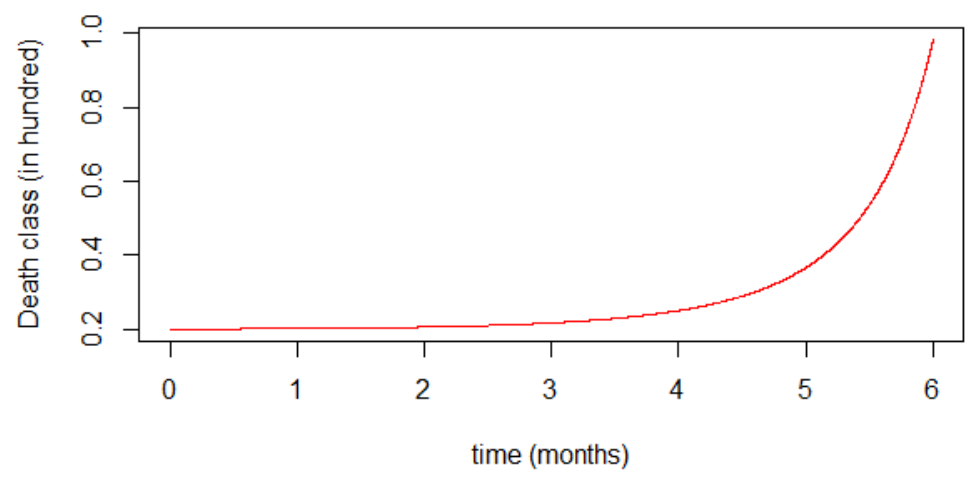

Figure 3. Variation of death class with time.

\section{Conclusion}

In this work, we have formulated a mathematical model that can be used to analyze the evolution of the Coronavirus. There exist unique solutions 
for the formulated system of ordinary differential equations. We have found four equilibrium points and all four equilibrium points are locally stable under some conditions. Global asymptotic stability of the recovered and death-free equilibrium point, infected and recovered free and death-free equilibrium point are also analyzed. The model parameters for the model were estimated using the Nelder-Mead optimization method. During the first 6 months, the infected population increases at a higher rate, the recovered population, and the death class increases at a lower rate. However, some modifications are needed. In the future, measures such as health precautions, vaccinations are needed to be considered for estimations of parameters in the model.

\section{Conflict of Interest}

The Authors declare no conflict of interest.

\section{References}

[1] M. C Anisiu, Lotka: Volterra and their model. Didact. Math. 2014, 32, 9-17.

[2] R. Bailey, T. J. Norman, B. Sendov; R. Tsanev. Mathematical models in biology and medicine. 1974.

[3] D. D. Neto, D. A. Teruel, K. Reichardt, D. R. Nielsen, J. A. Frizzone, O. O. S.Bacchi, Principles of crop modeling and simulation: I. Uses of mathematical models in agricultural science. Sci. Agric. 1998, 55, 46-50.

[4] S. M. H. Tabatabaie, J. P. Bolte, G. S. A. Murthy. Regional scale modeling framework combining biogeochemical model with life cycle and economic analysis for integrated assessment of cropping systems. Sci. Total Environ. 2018, $625,428-439$.

[5] G. P Pokhariyal, A. J . Rodrigues. An accurate epidemiological model. Appl. Math. Comput. 1993, 53, 1-12.

[6] H. W. Hethcote. Diseases. 2000, 42, 599-653.

[7] E. F. D. Goufo, R. Maritz, J. Munganga. Some properties of the KermackMcKendrick epidemic model with fractional derivative and nonlinear incidence. Adv. Differ. Equations. 2014, 2014, 278.

[8] R. M. Anderson, R. M. May. Helminth infections of humans: mathematical models, population dynamics, and control. Adv. Parasitol. 1985, 24, 1-101.

[9] L. E. Keshet. Mathematical models in biology. 2005.

[10] D. Baud, X. Qi, K. N. Saines, D. Musso, L. Pomar, G. Favre. Real estimates of 
mortality following COVID-19 infection. Lancet Infect. Dis. 2020, 20, 773.

[11] A. K. Misra, A. Sharma, J. B. Shukla. Modeling and analysis of effects of awareness programs by media on the spread of infectious diseases. Math. Comput. Model. 2011, 53(5-6), 1221-1228.

[12] A. Remuzzi, G. Remuzzi. COVID-19 and Italy: what next?. Lancet. 2020, 395, 1225-1228.

[13] Syed Inayat Ali Shah. A Dynamic compartmental mathematical model describing the transmissibility of MERS-CoV virus in public. J. Math. 2019, 51, 57-71

[14] R. U. Din, K. Shah, I. Ahmad, T. Abdeljawad. Study of transmission dynamics of novel COVID-19 by using mathematical model. Adv. Differ. Equations. 2020, 2020.

[15] Novel Coronavirus Information Center. Elsevier Connect. 2020. https://www.elsevier.com/connect/coronavirus-information-center

[16] A. Zeb, E. Alzahrani, V. S. Erturk, G. Zaman. Mathematical Model for Coronavirus Disease 2019 (COVID-19) Containing Isolation Class. Biomed Res. Int. 2020, 2020.

[17] K. Ramanathan. The COVID-19 resource centre - inc. 2020, 19-21.

[18] O. Zakary, S. Bidah, M. Rachik, H. Ferjouchia. Mathematical Model to Estimate and Predict the COVID-19 Infections in Morocco: Optimal Control Strategy. J. Appl. Math. 2020, 2020, 9813926.

[19] F. Ndaïrou, I. Area, J. J. Nieto, D. F. M. Torres. Mathematical modeling of COVID-19 transmission dynamics with a case study of Wuhan. Chaos, Solitons and Fractals. 2020, 135.

[20] H. Kınac1, M. G. Ünsal, R. A. Kasap. Close look at 2019 novel coronavirus (COVID 19) infections in Turkey using time series analysis \& efficiency analysis. Chaos, Solitons and Fractals. 2021, 143.

[21] A. Boudaoui, Y. E. Moussa, Z. Hammouch, S. A. Ullah. Fractional-order model describing the dynamics of the novel coronavirus (COVID-19) with nonsingular kernel. Chaos, Solitons and Fractals. 2021, 146.

[22] A. Zeroual, F. Harrou, A. Dairi, Y. Sun. Deep learning methods for forecasting COVID-19 time-Series data: A Comparative study. Chaos, Solitons and Fractals. 2020, 140, 110121.

[23] A. Kouidere, D. Kada, O. Balatif, M. Rachik, M. Naim. Optimal control approach of a mathematical modeling with multiple delays of the negative impact of delays in applying preventive precautions against the spread of the COVID-19 pandemic with a case study of Brazil and cost-effectiveness. Chaos, Solitons and Fractals. 2021, 142, 110438.

[24] K. S. Sharov. Creating and applying SIR modified compartmental model for 
calculation of COVID-19 lockdown efficiency. Chaos, Solitons and Fractals. 2020, 141, 110295.

[25] J. Farooq, M. A Bazaz. A novel adaptive deep learning model of Covid-19 with focus on mortality reduction strategies. Chaos, Solitons $\mathcal{E}$ Fractals. 2020, 138, 110148.

[26] S. Basu, R. H. Campbell. Going by the numbers: Learning and modeling COVID-19 disease dynamics. Chaos, Solitons \& Fractals. 2020, 138, 110140.

[27] J. K. K. Asamoah, M. A. Owusu, Z. Jin, F. T. Oduro, A. Abidemi, E. O. Gyasi. Global stability and cost-effectiveness analysis of COVID-19 considering the impact of the environment: using data from Ghana. Chaos, Solitons E Fractals. 2020, 140, 110103.

[28] M. S. Alqarni, M. Alghamdi, T. Muhammad, A. S. Alshomrani, M. A. Khan. Mathematical modeling for novel coronavirus (COVID-19) and control. Numer. Methods Partial Differ. Equ. 2020, 1-17.

[29] K. Lumpur. Mathematical Modeling of Coronavirus Reproduction Rate with Policy and Behavioral Effects Rabeya anzum, $1 \mathrm{Md}$. Zahidul Islam * 2 Introduction: Mathematical modeling of the any disease spreading is a very important tool to predict the Literature Review. Math. Model. Coronavirus Reprod. Rate with Policy Behav. Eff. 2020.

[30] O. D. Deborah. Mathematical Model for the Transmission of Covid-19 with Nonlinear Forces of Infection and the Need for Prevention Measure in Nigeria. J. Infect. Dis. Epidemiol. 2020, 6:158.

[31] S. H. A. Khoshnaw, R. H. Salih, S. Sulaimany. Mathematical modelling for coronavirus disease (COVID-19) in predicting future behaviours and sensitivity analysis. Math. Model. Nat. Phenom. 2020, 15, 1-13.

[32] R. Resmawan, L. Yahya. Sensitifity Analysis of Mathematical Model of Coronavirus Disease (COVID-19) Transmission. Cauchy. 2020, 6, 91-99.

[33] A. Dighe, T. Jombart, M. Kerkhove, N. A Ferguson. Mathematical model of the transmission of middle East respiratory syndrome coronavirus in dromedary camels (Camelus dromedarius). Int. J. Infect. Dis. 2019, 79, 1-150.

[34] Y. Belgaid, M. Helal, E. Venturino. Analysis of a Model for Coronavirus Spread. Mathematics. 2020, 8, 1-30.

[35] M. A. A. Oud, A. Ali, H. Alrabaiah, S. Ullah, M. A. Khan, S. A . Islam. Fractional order mathematical model for COVID-19 dynamics with quarantine, isolation, and environmental viral load. Adv. Differ. Equations. 2021, 2021, 106.

[36] M. Tomochi, M. A. Kono. Mathematical model for COVID-19 pandemic-SIIR model: Effects of asymptomatic individuals. J. Gen. Fam. Med. 2021, 22 (1), 5-14.

[37] B. Ivorra, M. R. Ferrández, M. V. Pérez, A. M. Ramos. Mathematical modeling of the spread of the coronavirus disease 2019 (COVID-19) considering its particular 
characteristics. The case of China. Commun. Nonlinear Sci. Numer. Simul. 2020, 88, 105303.

[38] C. Jost, R. Arditi. From pattern to process: identifying predator-prey models from time-series data. Popul. Ecol. 2001, 43, 229-243.

[39] S. R. Carpenter, K. L. Cottingham, C. A. Stow. Fitting predator-prey models to time series with observation errors. Ecology. 1994, 75, 1254-1264.

[40] C. Jost, R. Arditi. Identifying predator-prey processes from time-series. Theor. Popul. Biol. 2000, 57, 325-337.

[41] K. Das, M. N. Srinivas, V. Madhusudanan, S. Pinelas. Mathematical analysis of a prey-predator system: An adaptive back-stepping control and stochastic approach. Math. Comput. Appl. 2019, 24, 22. 